\title{
Representing the Richness of Avian Spatial Cognition: Properties of a Lateralized Homing Pigeon Hippocampus
}

\author{
Verner P. Bingman', Jennifer J. Siegel ${ }^{2}$, Anna Gagliardo ${ }^{3}$ and Jonathan T. Erichsen ${ }^{4}$ \\ 'Department of Psychology and J.P. Scott Center for Neuroscience, Mind and Behavior, \\ Bowling Green State University, Ohio, USA, ${ }^{2}$ Department of Neurobiology and Anatomy, \\ University of Texas Medical School at Houston, TX, USA, ${ }^{3}$ Dipartimento di Etologia, Ecologia ed Evoluzione, \\ Università di Pisa, Italy and ${ }^{4}$ Department of Optometry and Vision Sciences, Cardiff University, Wales, UK
}

\section{SYNOPSIS}

Brain organization and its relationship to behavior in any extant species is a reflection of a long evolutionary history of adaptive change. Therefore, it follows that the relationship between the hippocampus and spatial cognition in any species or taxonomic group would be characterized by features adapted to its spatial ecology. Birds are the animal world's supreme navigators, and aspects of their navigational ability are dependent on the integrity of the hippocampal formation. Using the homing pigeon as a model species, we review an accumulating body of data indicating that the avian hippocampus is functionally lateralized. The spatial response properties of left hippocampal neurons, as recorded in freely moving pigeons in a laboratory environment, differ from the response properties of right hippocampal neurons. Left hippocampal lesions generally disrupt navigational behavior under field conditions more than right lesions, while right lesions are more likely to disrupt goal localization behavior under laboratory conditions. We propose that the available data are consistent with a hypothesis of a left hippocampus more involved in navigational processes, and a right hippocampus more involved in representing the locations of events. We also discuss the extent to which the observed hippocampal lateralization

Reprint address:

Verner P. Bingman

Department of Psychology

Bowling Green State University

Bowling Green, $\mathrm{OH} 43403$, USA

e-mail: vbingma@bgnet.bgsu.edu should be viewed as an intrinsic property of the hippocampus itself or imposed by the lateralized properties of visual inputs originating in other brain regions. Whatever the nature of the observed hippocampal lateralization, it is likely one adaptive variation in hippocampal organization that supports the extraordinary spatial behavior of birds.

\section{KEY WORDS}

birds, cognitive map, hippocampal formation, navigation, spatial memory, unit recording

\section{INTRODUCTION}

The crucial role played by natural selection in shaping the organization of biological systems is an undeniable scientific truth. Almost any scientist would embrace this premise, yet in much of neuroscience its implications are often only perfunctorily acknowledged. However, the implications can be profound for research that examines the relationship between brain organization and the properties of neural representations that guide behavior, and highlight the importance of comparative research. Therefore, we begin with a set of statements about the role of natural selection in structuring brainbehavior relationships, and the implications of these assumptions for research into the functional organization of the amniote hippocampal formation (HF).

1. During evolution, natural selection has promoted, within a framework of genetic, developmental and other biological constraints, 
group-specific (e.g., species) adaptive changes in brain-behavior organization.

2. Therefore, differences in the selective pressures/ landscape of independently evolving groups should be associated with adaptive differences in brain-behavior organization.

3. Because all extant species have evolved independently for at least some period of time, it follows that the brain organization of any two species will differ across any number of dimensions as a consequence of group-specific adaptations.

The important message that emerges from these considerations is that there are opposing forces which will determine the extent to which brainbehavior organization between any two species resemble each other. On the one hand, taxonomic relatedness and shared evolutionary history, and the degree of similarity in the biological constraints associated with that relatedness, would impede divergence in taxonomically similar groups as well as oppose convergent similarity in more remotely related taxonomic groups. By contrast, shared selective landscapes would promote similarities even in groups that have evolved independently for a considerable period of time.

\section{The amniote hippocampal formation:} evolutionary/comparative considerations

The HF of reptiles, birds and mammals is a homologous structure that can be traced to the last common ancestor of these extant amniote groups some 300 million years ago. There is evidence to suggest that the range of cognitive processes thought to recruit participation of HF differs among amniote groups (contrast /4/ with /56/). However, the amniote HF plays a universally crucial role in the map-like representation of environmental/ landmark spatial relations (turtles $/ 41 /$; birds $/ 8$, $15 /$; rats $/ 12,36,45 /$; non-human primates $/ 36,45 /$; humans $/ 3 /$ ). The conserved role of the amniote HF in spatial cognition identifies the representation of space as the likely ancestral function of HF.

Despite the conserved role of HF in the maplike representation of space, a consideration of spatial ecology and behavior would suggest that the neuronal implementation of representing space should display adaptive variation among taxonomic groups. As perhaps an extreme example, the contrast between the extensively studied laboratory rat and homing pigeon could not be more striking. It is hard to imagine that HF-dependent spatial representations that support the diurnal, highly visual and open field spatial behavior of homing pigeons would share identical implementational mechanisms as those in the nocturnal and labyrinthine rat with relatively poor vision. It is not the intent of this review to summarize the vast literature on the properties of HF-dependent spatial behavior in the rat (see $/ 47 /$ for an excellent review). However, there are two features that are important for our discussion of the avian HF. First, any consideration of how the rat HF participates in implementing a representation of space begins with the acknowledged fundamental properties of socalled hippocampal place cells $/ 35 /$. Rat HF place cells are remarkably faithful in firing relatively few action potentials as a rat moves through a large portion of a test environment, but they display a dramatic increase in firing rate only when a rat passes through a specific, restricted portion of the test environment. Different place cells will be preferentially active in different portions of an environment. Second, to the best of our knowledge, there is no indication that the spatial response profile of rat place cells, nor the spatial processes controlled by place cells, varies between the HF of the left and right hemisphere.

\section{A LATERALIZED AVIAN HIPPOCAMPAL FORMATION}

\section{The right HF, synthesizing neuronal spatial response} profiles and lesion findings

With the rat $\mathrm{HF}$ place cell serving as background, a series of studies have been carried out to examine the spatial response profile of HF neurons (units) recorded from freely moving homing pigeons. For reasons unrelated to any expectation of possible lateralization, the first work recorded from the right $\mathrm{HF}$ only $/ 51 /$. There were two main conclusions that emerged from that study. First, none of the neurons displayed the same pattern of response profile typically associated with rat $\mathrm{HF}$ 
place cells. Specifically, although many right homing pigeon HF neurons displayed increases in firing rate in specific regions (patches) of the plusmaze test environment that were stable (reliable) across a recording session, the patches of higher activity generally had much lower temporal reliability and relatively smaller increases in firing rate above baseline compared to a typical rat $\mathrm{HF}$ place cell (e.g., Fig. 1D). In other words, a subject's location in space explained much less of the variance in firing rate of homing pigeon right $\mathrm{HF}$ cells than rat HF place cells. However, despite their relatively noisy spatial signal, many right HF cells displayed patches of higher activity at goal locations (food reward) placed at the terminal end of the plus-maze arms (e.g., Fig. IB). These neurons were not just responding to food, as many neurons would respond at only one or two of the four possible goal locations in the plus-maze and only one of four possible goal locations in a eightarm radial maze (see below $/ 28 /$ ). How might one characterize the response profile of these neurons? In our view, it is the interaction between location in space and the salience of some occurrence ("event") at that location which explains the spatial variation in firing rate. Said another way, the right HF neurons appear more responsive to the interaction between the momentary or transient occurrence of something biologically salient and location than location per se, the latter being more characteristic of rat place cells (but see $/ 27,34,65 /$ ).

Birds as a group are well adapted for remembering the location of events in space and using that spatial memory as a component of what might be called episodic memory to control their behavior in the future $17 \%$. Food storing birds that need to remember the locations of cached food are a notable example, and it is clear that memory for cache locations recruits participation of $\mathrm{HF} / 48$, 49/. Homing pigeons also readily recall the location of food seen 24 hours earlier, and HF lesions will compromise the ability of birds to use spatial cues to relocate that food $/ 55 /$. Therefore, the transient "event" quality of many right HF neurons and the robustness of episodic-like spatial memory for biologically salient experiences both lead to our working hypothesis that the right HF of homing pigeons, and other birds as well, is organized to crucially participate in representing what one might call "event locations".

Assuming our hypothesis approximates what is really occurring, to what properties of space might the right HF of birds be tuned? Hippocampal lesion data are of interest here. In chicks /58/ and homing pigeons $/ 29 /$, only right HF lesions were found to compromise the ability of birds to locate a goal location reliant on the geometric properties of the bounded experimental space or cues distributed distally in the experimental environment. Similarly, in an eye occlusion study the right hemisphere of the homing pigeon forebrain was concluded to be involved in only a global or geometric spatial reference system $/ 37 /$.

In summary, the lesion data suggest a right $\mathrm{HF}$ sensitive to global features of a test environment while the neuronal response data suggest a right HF sensitivity to locations of biological salience. Together, they promote the hypothesis that the right HF preferentially contributes to avian event (episodiclike) memory by participating in representing locations of both transient and stable biological salience relying on geometric or global properties of space.

\section{The left HF, synthesizing neuronal spatial response profiles and lesion findings}

Again for reasons unrelated to any expectation of possible lateralization, recordings from the left HF of homing pigeons were first carried out in an eight-arm radial maze $/ 28 /$. What emerged from this study was the discovery of a neuronal spatial response profile that was interesting from a perspective of navigation; a response property not found in the right HF. Rather than being preferentially active at goal locations in the test environment, neurons of this type, what we called "path cells", displayed increased firing rates along the corridors connecting goal locations. This work motivated a direct comparison of left and right $\mathrm{HF}$ neurons in the plus-maze environment (Fig. 1) /52/. Figure $1 \mathrm{~A}$ contains the rate map of one left $\mathrm{HF}$ path cell, which is recognized by the elongated regions of higher activity along the arms leading to the goal locations (in this case the north and south arms). Again, this type of spatial response profile has yet to be seen in the right HF. 

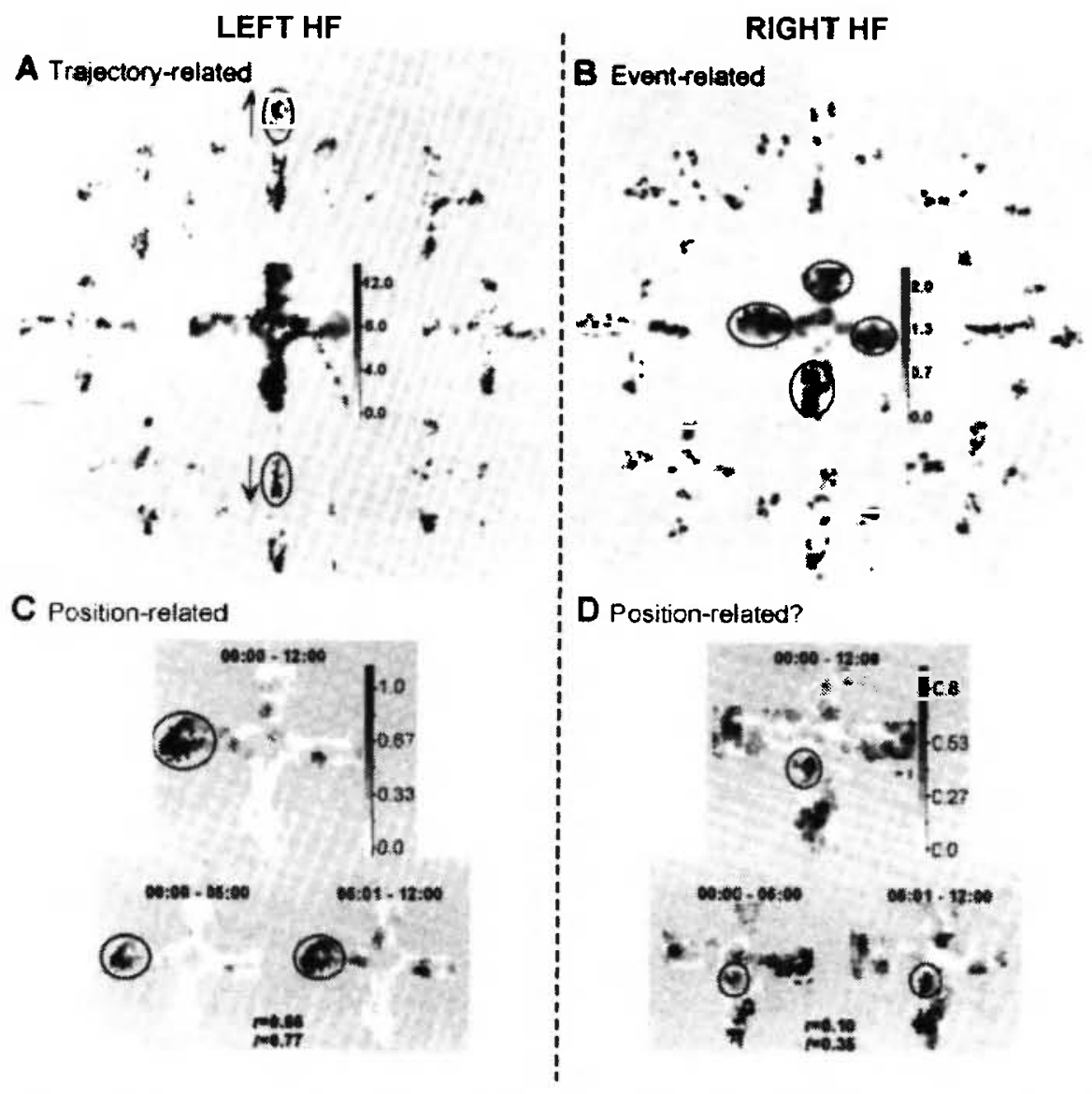

Fig. 1: Representative spatial response profiles of two left $(A, C)$ and two right (B, D) HF neurons recorded from homing pigeons as they moved through a plus-maze environment. Darker shades of grey represent locations in the plus maze with higher firing rates; lighter shades of grey lower firing rats. Approximate firing rates can be read by the accompanying scales (spikes/sec). Notable are the lateralized trajectory and event-associated activity related to a pigeon's momentary position in the plus maze. A, B. The larger, central plus maze represents the overall rate map while the smaller rate maps that surround it were generated only when a pigeon was moving in a particular direction (white arrows). The left HF cell in $\mathbf{A}$, a representative trajectory modulated unit, displayed higher activity when the pigeon was moving southward in the north arm (circled region and black arrow, bottom rate map), but did not display similar high activity when the pigeon moved northward in the same arm (circled region and black arrow, top rate map). A reverse of this pattern can be detected in the south arm. Right HF cells were much less likely to display trajectory-modulated activity (e.g., B, note the similarity in the distribution of firing rates across the directional rate maps). C, D. The larger plus maze at the top represents the overall rate map while the two smaller plus mazes below represent the rate maps generated during the first half (left) and second half (right) of the recording session. $r=$ rate map correlation coefficient for the two halves of the recording session; $I=$ overall spatial information score. Right, and to a lesser extent left, HF cells often displayed activity at the distal ends of maze arms where pigeons received food reward (B, C circled regions; D). Some cells displayed increased activity at all rewarded locations (e.g., B). Most cells appeared to display increased activity that was an interaction between reward and momentary position (i.e., increased activity at the ends of only a subset of maze arms; e.g., C, D). The circled patch of higher activity observed in the overall rate map of $\mathbf{D}$ was only observed during the second half of the recording session. The patch of higher activity observed in the west arm of $\mathbf{C}$ (circled), although exceptionally stable across the two halves of the recording session, was associated with a faster firing rate during the second half. Few cells in either the right or left $\mathrm{HF}$ displayed robust, reliable location-specific activity that was stable during the entire recording session of the type typically reported in the rodent Ammon's horn region. Rather, they generally displayed activity that appeared to be an interaction between location and additional behavioral conditions and/or events occurring in restricted regions of space. 
The path cells turn out to be even more interesting; the firing rates of these neurons are typically modulated by the direction a pigeon is moving. In Figure $1 \mathrm{~A}$, the smaller rate maps that surround the larger, overall rate map identify the spatial variation in firing rate when the pigeon was moving in one particular direction. For example, the smaller rate map on the top identifies spatial variation in firing rate associated with movement in a northerly (see black arrow) direction, while the smaller rate map on the bottom movement in a southerly (see black arrow) direction. What is immediately apparent is that the higher activity in the north arm of the maze occurred only when the pigeon was moving south exiting the arm but not when moving north entering it. Although not as striking, the directional pattern is reversed for the south arm; higher activity when moving north out of the arm than when moving south into it.

The directional analysis on the rate maps of several left $\mathrm{HF}$ path cells revealed a similar directional modulation of firing rate $152 \%$. What aspect of space may be represented by this firing rate profile? Location of the pigeon is insufficient because higher activity is observed at locations only when a pigeon is moving in a limited range of directions. Direction is also insufficient; some locations are associated with higher firing rates when a pigeon is moving in one direction, while other locations are associated with higher firing rates when movement is in another direction (e.g., Fig. 1A). Thus it is an interaction between location and direction that best explains the response profile of the path cells. This interaction suggests a role in the computation of a navigational trajectory prospectively related to where a pigeon is going, retrospectively related to where it is coming from, or some combination of the two (see /13/).

It should be noted that, in addition to path cells, neurons that preferentially fire at goal locations are also found in the left HF. The neuron displayed in Figure 1C is extraordinary because of its welldefined patch of higher activity in the west goal location and the reliability $(0.66)$ of that patch over the course of the recording session. By any standard, the spatial response profile of this neuron has the quality of a bona fide rat place cell. How numerous such, up to now, rare neurons are in the homing pigeon $\mathrm{HF}$ remains undetermined, but from the perspective of the left $\mathrm{HF}$ and navigational computation, it is curious that even the activity of this left neuron was modulated by the direction of movement.

The hypothesis that emerges from the unit data is a left HF preferentially involved in the computation of a navigational trajectory. In support of this idea is that left but not right HF lesions can impair navigational map learning in homing pigeons $/ 16 /$, and that this learning deficit can be explained by a preferential role of the left $\mathrm{HF}$ in representing (associating) the directional relationship among environmental stimuli and a goal location(s) using the sun compass /14/. Summarized in Figure $2 \mathrm{~A}$ is the performance of a group of control, right $\mathrm{HF}$ and left HF lesioned pigeons on a task that tested their ability to learn a goal direction in an outdoor, octagonal arena with their sun compass. All three groups learned the task, but when subsequently tested after being subjected to a phase-shift of the light-dark cycle, only the control and right $\mathrm{HF}$ lesioned pigeons displayed a shift in orientation indicative of a sun compass directional representation. After phase-shift, the left HF lesioned pigeons as a group were generally disoriented and gave no indication that they were able to learn a sun compass-based directional representation of the goal. For birds, the sun compass is the prevailing spatial behavior mechanism that will guide orientation in space. In the field with the position of the sun discernable, the output of any navigational computation, whether HF dependent or not, will be a sun compass-guided bearing. Sun compass-based spatial learning deficits following left HF lesion only are consistent with the hypothesis of preferential involvement of the left $H F$ in the computation of navigational trajectories based on the unit profile data.

Although not tests of HF function, there are several eye occlusion studies that are relevant to a discussion of a left HF preferentially involved in navigating among goal locations. Right eye (feeding into the left forebrain hemisphere) occlusions have been consistently found, albeit modestly, to compromise homing performance in the field $/ 11$, 38,60 . In a songbird species, migratory orientation based on the Earth's magnetic field is also 


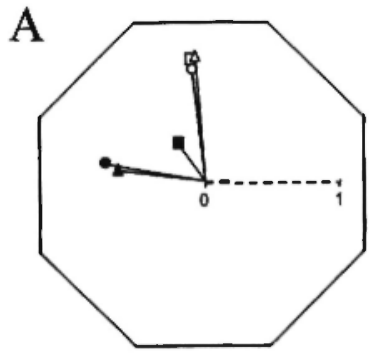

\section{Training and Test after Clock-shift without Visual Features}

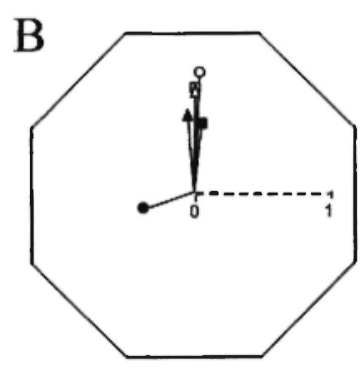

Training and Test after Clock-shift with Visual Features

Fig. 2: A. Summary of the directional choices of control, left and right HF lesioned homing pigeons on a sun compass-based learning task in the absence of visual feature cues during the last day of training (vectors with open heads: circle, control; square, left HF lesioned; triangle, right HF lesioned) and during the test session following exposure to a phase shift of the light-dark cycle (vectors with filled heads). The training direction is normalized across individuals to the top of the octagonal area. Group mean-vector lengths can be read by the scale indicated by the hatched line in the diagram. All groups successfully learned the task but only the control and right HF lesioned pigeons displayed the shift in directional choices during test indicative of learning a sun compass representation of the goal location. B. Summary of the directional choices (group mean vectors) of control, left and right HF lesioned pigeons on a sun compass-based learning task in the presence of similarly predictive visual feature cues (see $\mathbf{A}$ for an explanation of the remainder of the diagram). Note that while control pigeons continue to show a shift in directional choices during test, indicative of sun compass use, the right $\mathrm{HF}$ lesioned pigeons no longer do so.

disrupted by right but not left eye occlusion /64/. The apparent right eye-left hemisphere bias in the control of navigational components of homing and migration lends further support to the hypothesis of a left HF preferentially involved in computing navigational trajectories.

\section{A LATERALIZED HOMING PIGEON HIPPOCAMPUS: INTRINSIC OR IMPOSED PROPERTY?}

Vision being the predominant sense in birds for guiding most behaviors, including components of spatial navigation, it is important to recognize that the avian visual system itself displays an evident lateralization of function (see below). Therefore, the question naturally arises whether any aspects of the lateralization of the HF merely reflect or are even a direct consequence of such properties of the visual system, which after all is providing a major sensory input for spatial navigation.

As is typical of many non-mammalian vertebrates generally, and in particular those with laterally placed eyes, birds have a fully crossed visual system. That is, the entire visual input from each eye projects to the contralateral side of the brain. The complete decussation (at least at this level) reflects the relatively small binocular overlap between the visual fields of the two eyes in most species of birds (predatory birds such as hawks and owls would be exceptions) and suggests that the two halves of the visual system are somewhat independent of each other. It is precisely this arrangement in birds that underlies and enables the various monocular (eye occlusion) experiments used to investigate hemispheric lateralization, and by inference HF lateralization. Although some inter-hemispheric transfer of monocularly obtained visual information does occur, the extent of such "inter-ocular" transfer is often quite limited and clearly varies dramatically with the nature of the stimuli and/or the portion of the retina used during a discrimination task as well as with the visually guided behavior used to respond $/ 18,63 /$.

Hemispheric specialization had been thought to be restricted to humans and some non-human primates until a variety of studies of nonmammalian vertebrates began to reveal behavioral signs of significant brain asymmetries $/ 26 /$. Early work by Andrew et al. $/ 1 /$, among others, provided the first clear evidence for such lateralization in birds. In particular, studies of visually guided behavior demonstrated an advantage (or dominance) of one eye over the other for different tasks. 
For example, in an operant discrimination task, pigeons displayed a consistent increase in correct pecking responses when choosing between two stimuli using the right eye (e.g. /19/), a finding consistent with other early studies in chicks and pigeons, which enjoyed a higher success rate when feeding with the right eye $124,43 \%$. A variety of subsequent experiments have reinforced the conclusion of "handedness" in birds, but just as in the case of interocular transfer studies, the preferred eye depends on the exact experimental conditions, including the characteristics of the stimuli as well as the visually guided motor response made by the bird $/ 59 /$.

The anatomical basis of visual lateralization has received a great deal of attention within the last decade or so, with most of the pathway analysis having been carried out in the pigeon and, to a lesser extent, in the chick. As in humans, a number of morphological asymmetries have been discovered, including: (1) larger superficial tectal cells on the left but larger neurons in laminae 13-15 on the right $/ 21 / ;(2)$ an asymmetry in commissural (interhemispheric) connections that leads to the left nucleus rotundus (i.e. in the dominant hemisphere) receiving input from both eyes while the right rotundus mainly represents only the left eye $/ 25 /$; (3) asymmetric tectotectal modulation via commissural connections $131 /$; (4) a greater asymmetry in the number of thalamic cells projecting to the ipsiand contralateral Wulst, which seems to be established by light exposure early in embryonic development $/ 10,32,44 /$; and (5) even qualitative differences in the information processed by the two (tectofugal and thalamofugal) pathways $/ 9 /$. Interestingly, however, no morphological differences have been reported in the retinas of the two eyes 122 . It is also noteworthy that, although there is considerable support for performance asymmetries in the avian visual system, the visual acuities of the two eyes appear to be virtually identical in pigeons 123/. However, the important point is that there appear to be numerous anatomical substrates or correlates for the observed behavioral asymmetry in visual performance. In the future, these and many other structures might be manipulated to investigate lateralization of the avian visual system and in turn its impact on the lateralization of the HF.
It is outside the scope of this paper to consider the extensive literature that has arisen as a result of investigations into the functional importance of visual lateralization (for reviews see $/ 20,61 /$ ), and there is the added potential complication that not all avian species, e.g. pigeons and chicks, may share exactly the same form of brain asymmetries. However, it is possible to attempt some generalized conclusions about the specialized functional abilities of each eye (or its contralateral brain hemisphere) in the hope that we might be able to consider the observed lateralization of the HF in light of the properties of the avian visual system. In brief, there is basic agreement that visual object processing, including pattern discrimination, is preferentially located in the left hemisphere $/ 20,22$, $31,42 /$, whereas the right hemisphere is associated with enhanced performance in responses guided by emotionally charged/salient stimuli as well as spatial, positional and topological cues $139,40,61$, 62\%. The combination of a sensitivity to stimulus salience and spatial cues would seem to fit nicely our conceptualization of a right $\mathrm{HF}$ involved in representing spatial components of episodic memory. In addition, experiments in chicks indicate that the left hemisphere is favored when a motor plan or response needs to be established and carried out, whereas the right hemisphere is employed to process topographical information $/ 2,57 \%$. Interestingly, when homing pigeons are released with one eye covered, their initial orientation (and subsequent superior performance) was significantly better when using the right eye (left hemisphere), and it was suggested that this reflects an inherent lateralized sensitivity to optic flow /38/. In any event, this result is consistent with the right eye's proposed superiority in carrying out a motor plan in the chick, and is consistent with the hypothesis that the left HF is preferentially involved in navigational processes in homing pigeons.

\section{COMPLEX SPATIAL BEHAVIOR AND THE INTEGRATION OF LATERALIZED HIPPOCAMPAL ELEMENTS}

Regardless of the extent to which $\mathrm{HF}$ lateralization in birds is an intrinsic property of an as yet unidentified asymmetry of neurobiological 
origin or a consequence of lateralized forebrain inputs, the role of HF in spatial cognition is asymmetrically organized in the two hemispheres. However, realization of a functionally lateralized HF carries with it the risk of viewing the two HFs as independent spatial processing elements, which they clearly are not. In our opinion, the most important organizational principle that shapes avian $\mathrm{HF}$ function is that the two HFs are integrated in determining the properties of spatial memory and the navigational mechanisms dependent on that memory. In fact, the computational range of the avian $\mathrm{HF}$, and its importance for spatial cognition, may be much more than the sum of elemental properties that are asymmetrically organized in the two forebrain hemispheres.

The importance of integration is apparent when considering that there is a large anatomical pathway, via the hippocampal commissure, that reciprocally connects the left and right HF $/ 5 /$. Whatever aspects of space that preferentially recruit participation of the left $\mathrm{HF}$ will also influence operation of the right $\mathrm{HF}$, as the right will influence the left HF. Left and right HF functional organization may be partially dissociable experimentally, but the asymmetrical functional descriptions that come from lateralization studies should be viewed as a corruption of emergent functional properties of the integrated system. Therefore, it is not surprising that when homing pigeons are challenged with navigating over a familiar landscape $150 /$, or blackcapped chickadees (Poecile atricapillus) with recalling the location of hidden food $/ 54 /$, immediate early gene activation reveals increased neuronal activity in the HFs of both hemispheres. Right and left $\mathrm{HF}$ lesions similarly interfere with the ability of homing pigeons to rely on a map-like representation of familiar landmarks to navigate to their home loft $/ 17 /$. To the best of our knowledge, only Sadananda and Bischof $/ 46 /$, in a study of sexual imprinting in zebra finches (Taeniopygia guttata), reported an asymmetry in HF recruitment in the control of a naturally occurring behavior.

The sometimes subtle importance of the interaction between the left and right HF hemisphere in the control of behavior is highlighted by the sun compass-based spatial learning task summarized in Figure 2A /14/. Recall that only left HF lesions were found to disrupt this learning. However, the pattern of results becomes much more complex if pigeons are trained to find the goal location with their sun compass in the presence of conspicuous feature cues that also identify the goal location. Again, control, left HF- and right HF-lesioned birds learned the task equally well (Fig. 2B). When subsequently tested following a phase shift of the light-dark cycle, control pigeons showed a corresponding shift in their directional choices demonstrating that, even in the presence of feature cues, they preferentially rely on a sun compass representation of the goal location. The pigeons with left HF lesions, the treatment that led to disorientation following phase shift of the lightdark cycle in the absence of feature cues, continued to orient in the correct training direction in accordance with the feature cues. The interesting group was the pigeons with right $\mathrm{HF}$ lesions, which were shown to be capable of learning a sun compass representation. In this experiment, rather than using the sun compass like the controls, the right $\mathrm{HF}$ lesioned pigeons as a group continued to orient in the training direction based on the feature cues. The conclusion that follows from this finding is that even though right $\mathrm{HF}$ lesions were found not to impair learning a sun compass representation of a goal location (Fig. 2A, experiment without feature cues), an intact right HF was necessary to support a left HF-mediated, preferential use of a sun compass representation over alternative feature cues. In other words, right HF lesions tended to reverse the hierarchical relationship between sun compass versus feature cue goal representational strategies. Whatever it is about the interaction between the left and right HF that enables the ascendancy of a sun compass representation is a mystery. However, it is hard to imagine that a working dichotomy of a navigational left $\mathrm{HF}$ and an event-sensitive right $\mathrm{HF}$ offers an adequate explanation. We have much to learn about the synergistic consequences of interactions between the left and right avian HF in the control of spatial cognition. 


\section{OF BIRDS AND MEN: A CURIOUS COMPARATIVE NOTE}

Unit recording and lesion data taken from homing pigeons and chicks, as well as indirectly eye occlusion data in songbirds $/ 6 /$, collectively lead to the conclusion that HF functional lateralization is a general property of avian forebrain organization. In fact, it is not a difficult experimental challenge to reveal the influence of leftright differences in $\mathrm{HF}$ on spatial behavior; although it certainly remains a challenge to offer a coherent functional characterization of the empirically observed differences. Therefore, it is interesting that, in the laboratory rat and nonhuman primates, experimental indications of $\mathrm{HF}$ functional lateralization are to the best of our knowledge virtually non-existent (but see $/ 30 /$ ). This is not to say that the HF of the laboratory rat and non-human primates is not functionally lateralized, only that any lateralization is not nearly as experimentally demonstrable as it is in birds. Indeed, the only vertebrate group in which $\mathrm{HF}$ functional lateralization is as easily demonstrable as it is in birds is humans.

Characterizing the left-right differences in the human HF is no easier than it is in birds, but there was a first indication from studying people with unilateral HF damage that the right HF may play a more substantial role in spatial cognition $/ 53 /$. More recent neuropsychological and brain imaging studies have revealed a pattern of differential deficits and asymmetrical patterns of neuronal activation suggesting that the right $\mathrm{HF}$ is more involved in processes of navigation, with the left HF being more involved in aspects of episodic memory $/ 3 /$. These hemispheric differences should not be viewed as a discrete functional partitioning, but biases that weigh the HF of one hemisphere more heavily than the other for some types of representational challenges.

What is truly curious is the language researchers have used to characterize the aspects of cognition that are suspected to lateralize. We proposed a navigational left $\mathrm{HF}$ and an event, episodic-like memory right $\mathrm{HF}$ asymmetry in homing pigeons without knowing that a "navigational" right and "episodic" left HF bias had already been proposed in humans. The HF lateralization observed in birds and humans is likely to be an example of convergent evolution in brain organization because there are no shared taxonomic groups that show a similar pattern of lateralization, at least as nearly robustly, and the current conceptualizations of what lateralizes are reversed in the two groups (e.g., the navigational $\mathrm{HF}$ is on the left in birds and the right in humans). This raises the question of the selective forces that promoted HF lateralization in birds and humans. Without drifting too far into pure speculation (some would argue it is already too late!) it seems likely that the evolution of HF lateralization in birds is in part related to selective pressure(s) enhancing the richness of HF-mediated spatial representations used for navigation and memory. Indeed, HF functional lateralization likely operates together with hypertrophied pallial regions of the avian forebrain to explain some of the extraordinary cognitive abilities of birds. In man, one cannot help suspect that the evolution of language and language as a representational device for the encoding of memory substantially contributed to the evolution of HF lateralization.

\section{SUMMARY}

The often spectacular navigational and spatial memory ability of birds as a group has evolved in parallel with a brain organization capable of supporting the sensory, motor and cognitive demands associated with these abilities. We have proposed that a lateralized HF is one adaptive property of avian brain organization that evolved in support of the complexity and richness of spatial cognition in birds. However, this generalization should be viewed with the recognition that different avian species will likely show variations in the general pattern of lateralization consistent with the selective forces associated with a particular species spatial ecology. Homing pigeons and chicks may be useful general models of avian HF organization, but adaptive, species-specific variation is to be expected. Differences in HF volume that correlate with spatial ecology would be one example of species-specific variation (e.g. /33/). Finally, HF is embedded in a complex network of interacting brain regions that collectively enable memory representations used to guide spatial behavior. 
Therefore, HF functional lateralization is only one element of an avian brain organization whose collective functional properties produce the richness of bird behavior.

\section{ACKNOWLEDGEMENTS}

This paper was written while VPB was supported as a Leverhulme Visiting Professor and BBSRC International Fellow at Cardiff University, Wales, UK. Support for some of the research described in this paper has been generously provided by the National Science Foundation (IBN0075891) and NATO.

\section{REFERENCES}

1. Andrew RJ, Mench J, Rainey C. Right-left asymmetry of response to visual stimuli in the domestic chick. In: Ingle DI, Goodale MA, Mansfield R, eds. Advances in Analysis of Visual Behavior. Cambridge, MA: MIT Press, 1982; 197-209.

2. Andrew RJ, Tommasi L, Ford N. Motor control by vision and the evolution of cerebral lateralization. Brain Lang 2000; 73: 220-235.

3. Burgess N, Maguire EA, O'Keefe J. The human hippocampus and spatial and episodic memory. Neuron 2002; 35: 625-621.

4. Bunsey M, Eichenbaum H. Conservation of hippocampal memory function in rats and humans. Nature 1996; 379: 255-257.

5. Casini $G$, Bingman VP, Bagnoli $P$. Connections of the pigeon dorsomedial forebrain studied with WGA-HRP and $\left({ }^{3} \mathrm{H}\right)$-proline. J Comp Neurol 1986; 245: 454-470.

6. Clayton NS. Lateralization and unilateral transfer of spatial memory in marsh tits. J Comp Physiol A 1993; 171: 799-806.

7. Clayton NS, Dickinson A. Scrub jays (Aphelocoma coerulescens) remember the relative time of caching as well as the location and content of their caches. Behav Neurosci 1999; 113: 403-416.

8. Colombo $\mathrm{M}$, Broadbent $\mathrm{N}$. Is the avian hippocampus a functional homologue of the mammalian hippocampus? Neurosci Biobehav Rev 2000; 24: 465-484.

9. Deng C, Rogers LJ. Differential contributions of the two visual pathways to functional lateralization in chicks. Behav Brain Res 1997; 87:173-182.

10. Deng $C$, Rogers LJ. Prehatching visual experience and lateralization in the visual Wulst of the chick. Behav Brain Res 2002;134:375-385.

11. Diekamp B, Prior H, Ioale P, Odetti F, Güntürkün $O$, Gagliardo A. Effects of monocular viewing on orientation in an arena at the release site and homing performance in pigeons. Behav Brain Res 2002; 136 : 103-111.

12. Eichenbaum H, Stewart C, Morris RGM. Hippocampal representation in place learning. J Neurosci 1990; 10: 3531-3542.

13. Ferbinteanu J, Shapiro ML. Prospective and retrospective memory coding in the hippocampus. Neuron 2003; 40: 1227-1239.

14. Gagliardo A, Bingman VP, Vallortigara G. Hippocampal lateralization of sun compass-based spatial learning in homing pigeons. $4^{\text {th }}$ FENS, Lisbon, July $10-$ 14, FENS Abst 2004; vol. 2, Al12.23.

15. Gagliardo A, Ioalé $\mathrm{P}$, Bingman VP. Homing in pigeons: the role of the hippocampal formation in the representation of landmarks used for navigation. J Neurosci 1999; 19: 311-315.

16. Gagliardo A, Ioale P, Odetti F, Bingman VP, Siegel JJ, Vallortigara $\mathrm{G}$. Hippocampus and homing pigeons: left and right hemispheric differences in navigational map learning. Eur J Neurosci 2001; 13: 1-9.

17. Gagliardo A, Odetti F, Ioale P, Bingman VP, Tuttle S, Vallortigara G. Bilateral participation of the hippocampus in familiar landmark navigation by homing pigeons. Behav Brain Res 2002; 136: 201-209.

18. Gaston KE. Interocular transfer of pattern discrimination learning in chicks. Brain Res 1984; 310: 213-221.

19. Güntürkün $O$. Lateralization of visually controlled behavior in pigeons. Physiol Behav 1985; 34: 575-577.

20. Güntürkün O. Avian visual lateralization: a review. NeuroReport 1997; 8: iij-xi.

21. Güntürkün $\mathrm{O}$. Morphological asymmetries of the tectum opticum in the pigeon. Exp Brain Res 1997; 116: 561 566.

22. Güntürkün $O$. Visual lateralization in birds: From neurotrophins to cognition? Eur J Morphol 1997; 35: 290-302.

23. Güntürkün $O$, Hahmann U. Visual acuity and hemispheric asymmetries in pigeons. Behav Brain Res 1994; 60: 171-175.

24. Güntürkün $\mathrm{O}$, Kesch $\mathrm{S}$. Visual lateralization during feeding in pigeons. Behav Neurosci 1987; 101: 433435.

25. Güntürkün $O$, Hellmann $B$, Melsbach $G$, Prior $H$. Asymmetries of representation in the visual system of pigeons. NeuroReport 1998; 9: 4127-4130.

26. Harris JA, Guglielmotti V, Bentivoglio M. Diencephalic asymmetries. Neurosci Biobehav Rev 1996; 20: 637-643

27. Hollup SA, Molden S, Donnett JG, Moser MB, Moser El. Accumulation of hippocampal place fields at the goal location in an annular watermaze task. J Neurosci 2001; 21: 1635-1644.

28. Hough GE $2^{\text {nd }}$, Bingman VP. Spatial response properties of homing pigeon hippocampal neurons: correlations with goal locations, movement between goals, and environmental context in a radial-arm arena. J Comp Physiol A 2004; 190: 1047-1062. 
29. Kahn MC, Bingman VP. Lateralization of spatial learning in the avian hippocampal formation. Behav Neurosci 2004; 118: 333-344.

30. Kawakami R, Shinohara $Y$, Kato $Y$, Sugiyama $H$, Shigemoto R, Ito I. Asymmetrical allocation of NMDA receptor e2 subunits in hippocampal circuitry. Science 2003; 300: 990-994.

31. Keysers C, Diekamp B, Gunturkun B. Evidence for physiological asymmetries in the intertectal connections of the pigeon (Columba livia) and their potential role in brain lateralisation. Brain Res 2000; 852: 406-413.

32. Koshiba M, Nakamura S, Deng C, Rogers LJ. Lightdependent development of asymmetry in the ipsilateral and contralateral thalamofugal visual projections of the chick. Neurosci Lett 2003; 336: 81-84.

33. Krebs JR, Sherry DF, Healy SD, Perry VH, Vaccarino AL. Hippocampal specialization of food-storing birds. Proc Natl Acad Sci USA 1989; 86: 1388-1392.

34. Lenck-Santini P-P, Save E, Poucet B. Evidence for a relationship between place-cell spatial firing and spatial memory performance. Hippocampus 2001 ; 11 : 377 390.

35. O'Keefe J, Dostrovsky J. The hippocampus as a spatial map: preliminary evidence from unit activity in the freely moving rat. Brain Res 1971 ; 34: 171-175.

36. Parkinson JK, Murray EA, Mishkin M. A selective mnemonic role for the hippocampus in monkeys: memory for the location of objects. J Neurosci 1988; 8: 4159-4167.

37. Prior $H$, Lingenauber $F$, Nitschke J, Güntürkün $O$. Orientation and lateralized cue use in pigeons navigating a large indoor environment. J Exp Biol 2002; 205: 1795-1805.

38. Prior $H$, Wiltschko $R$, Stapput $K$, Güntürkün $O$, Wiltschko, W. Visual lateralization and homing in pigeons. Behav Brain Res 2004; 154: 301-310.

39. Rashid N, Andrew RJ. Right hemisphere advantage for topographical orientation in the domestic chick. Neuropsychologia 1989; 27: 937-948.

40. Regolin L, Garzotto B, Rugani R, Pagni P, Vallortigara G. Working memory in the chick: parallel and lateralized mechanisms for encoding of object- and position-specific information. Behav Brain Res 2005; 157: 1-9.

41. Rodriguez F, Lopez JC, Vargas, JP, Gomez, Y, Broglio $\mathrm{C}$, Salas $\mathrm{C}$. Conservation of spatial memory function in the pallial forebrain of reptiles and ray-finned fishes. J Neurosci 2002; 22: 2894-2903.

42. Rogers LJ. Behavioral, structural and neurochemical asymmetries in the avian brain: a model system for studying visual development and processing. Neurosci Biobehav Rev 1996; 20: 487-503.

43. Rogers LJ, Anson JM. Lateralisation of function in the chicken fore-brain. Pharmacol Biochem Behav 1979; 10: 679-686.

44. Rogers LJ, Sink HS. Transient asymmetry in the projections of the rostral thalamus to the visual hyper- striatum of the chicken, and reversal of its direction by light exposure. Exp Brain Res 1988; 70: 378-384.

45. Rolls ET Spatial view cells and the representation of place in the primate hippocampus. Hippocampus 1999; 9: 467-480.

46. Sadananda M, Bischof H-J. c-fos is induced in the hippocampus during consolidation of sexual imprinting in the zebra finch (Taeniopygia guttata). Hippocampus 2004; 14: 19-27.

47. Sharp PE. The Neural Basis of Navigation: Evidence from Single Cell Recording. Boston, MA: Kluwer Academic, 2002.

48. Sherry DF, Vaccarino AL. Hippocampus and memory for food caches in black-capped chickadees. Behav Neurosci 1989; 103: 308-318.

49. Shiflett MW, Smulders TV, Bebedict L, DeVoogd TJ. Reversible inactivation of the hippocampal formation in food storing black-capped chickadees (Poecile atricapillus). Hippocampus 2003; 13: 437-444.

50. Shimizu T, Bowers AN, Budzynski C, Kahn MC, Bingman VP. What does a pigeon brain look like during homing? Selective examination of ZENK expression in the telencephalon of pigeons navigating home. Behav Neurosci 2004; 118: 845-851.

51. Siegel JJ, Nitz D, Bingman VP. Spatial-specificity of single-units in the hippocampal formation of freely moving homing pigeons. Hippocampus 2005; 15: 2640.

52. Siegel JJ, Nitz D, Bingman VP. Lateralized functional components of spatial cognition in the avian hippocampal formation: evidence from single-unit recordings in freely moving homing pigeons. Hippocampus; in press.

53. Smith ML, Milner B. Right hippocampal impairment in the recall of spatial location: Encoding deficit or rapid forgetting? Neuropsychologia 1989; 27: 71-81.

54. Smulders TV, DeVoogd TJ. Expression of immediate early genes in the hippocampal formation of the blackcapped chickadee (Poecile atricapillus) during a foodhoarding task. Behav Brain Res 2000; 114: 39-49.

55. Strasser R, Bingman VP. The effects of hippocampal lesions in homing pigeons on a one-trial food association task. J Comp Physiol A 1999; 185: 583-590.

56. Strasser R, Ehrlinger J, Bingman VP. Transitive behavior in hippocampal-lesioned homing pigeons. Brain Behav Evol 2004; 63: 181 -188.

57. Tommasi L, Andrew RJ. The use of viewing posture to control visual processing by lateralised mechanisms. J Exp Biol 2002; 205: 1451-1457.

58. Tommasi L, Gagliardo A, Vallortigara G. Separate processing mechanisms for encoding of geometric and landmark information in the avian hippocampus. Eur $\mathrm{J}$ Neurosci 2003; 17: 1695-1702.

59. Tommasi L, Andrew RJ, Vallortigara G. Eye use in search is determined by the nature of task in the dornestic chick (Gallus gallus). Behav Brain Res 2000; 112: 119-126. 
60. Ulrich C, Prior H, Duka T, Leshchins'ka I, Valenti P, Güntürkün $O$, Lipp H-P. Left-hemispheric superiority for visuospatial orientation in homing pigeons. Behav Brain Res 1999; 104: 169-178.

61. Vallortigara G. Comparative neuropsychology of the dual brain: a stroll through animals' left and right perceptual worlds. Brain Lang 2000; 73: 189-219.

62. Vallortigara G, Regolin L, Bortolomiol G, Tommasi L. Lateral asymmetries due to preferences in eye use during visual discrimination learning in chicks. Behav Brain Res 1996; 74: 135-143.
63. Watanabe S. Interocular transfer of learning in the pigeon: visuo-motor integration and separation of discriminanda and manipulanda. Behav Brain Res 1986; 19: 227-232.

64. Wiltschko $W$, Traudt J, Güntürkün $\mathrm{O}$, Prior $\mathrm{H}$, Wiltschko $R$. Lateralization of magnetic compass orientation in a migratory bird. Nature 2002 ; 419 : 467 470.

65. Wood ER, Dudchenko PA, Robitsek RJ, Eichenbaum $H$. Hippocampal neurons encode information about different types of memory episodes occurring in the same location. Neuron 2000; 27: 623-633. 